\title{
FDTD method and models in optical education
}

Xiaogang Lin, Nan Wan, Lingdong Weng, Hao Zhu, Jihe Du

Xiaogang Lin, Nan Wan, Lingdong Weng, Hao Zhu, Jihe Du, "FDTD method and models in optical education," Proc. SPIE 10452, 14th Conference on Education and Training in Optics and Photonics: ETOP 2017, 104524J (16 August 2017); doi: 10.1117/12.2268324

SPIE Event: 14th Conference on Education and Training in Optics and Photonics, ETOP 2017, 2017, Hangzhou, China 


\title{
FDTD Method and Models in Optical Education
}

\author{
Xiaogang Lin*, Nan Wan, Lingdong Weng, Hao Zhu and Jihe Du \\ Key Laboratory of Optoelectronic Technology and Systems of Ministry of Education \\ of China, Chongqing University, Chongqing, 400044, China
}

\begin{abstract}
In this paper, finite-difference time-domain (FDTD) method has been proposed as a pedagogical way in optical education. Meanwhile, FDTD solutions, a simulation software based on the FDTD algorithm, has been presented as a new tool which helps abecedarians to build optical models and to analyze optical problems. The core of FDTD algorithm is that the time-dependent Maxwell's equations are discretized to the space and time partial derivatives, and then, to simulate the response of the interaction between the electronic pulse and the ideal conductor or semiconductor. Because the solving of electromagnetic field is in time domain, the memory usage is reduced and the simulation consequence on broadband can be obtained easily. Thus, promoting FDTD algorithm in optical education is available and efficient. FDTD enables us to design, analyze and test modern passive and nonlinear photonic components (such as bio-particles, nanoparticle and so on) for wave propagation, scattering, reflection, diffraction, polarization and nonlinear phenomena. The different FDTD models can help teachers and students solve almost all of the optical problems in optical education. Additionally, the GUI of FDTD solutions is so friendly to abecedarians that learners can master it quickly.
\end{abstract}

Keywords: FDTD method, FDTD solutions, optical education, electromagnetic field

\section{Introduction}

Electromagnetic theory has been proposed, developed and applied in the past one hundred years. The solutions to solve electromagnetic problems as well as Maxwell equations also have been developed accompanying the development of computional technology, such as Method of Moment (MOM), Finite Element Method (FEM), Boundary Element Method (BEM) and Finite Difference Time Domain (FDTD) method. Dating back to the year of 1966, Yee has proposed the FDTD method, which is his particular discrete scheme for Maxwell's equations ${ }^{[1]}$. In 1969, Taylor proposed the absorbing boundary to absorb the outward traveling wave ${ }^{[2]}$. In 1975, Taflove discussed the near-far field transformation and the numerical stability ${ }^{[3]}$. In 1981, Mur used the first order and the second order boundary condition to solve the problem of absorbing at the boundary ${ }^{[4]}$. In 1987, Kasher and Yee

14th Conference on Education and Training in Optics and Photonics: ETOP 2017, edited by Xu Liu,

Xi-Cheng Zhang, Proc. of SPIE Vol. 10452, 104524J - (o 2017 ICO, IEEE, OSA, SPIE

CCC code: $0277-786 \mathrm{X} / 17 / \$ 18 \cdot$ doi: $10.1117 / 12.2268324$

Proc. of SPIE Vol. 10452 104524J-1 
proposed the subgridding method ${ }^{[5]}$. In 1994, Berenger proposed the Perfectly Matched Layer (PML) boundary condition ${ }^{[6]}$. And the PML boundary conditions has been developed and applied in solving electromagnetic problems until now.

After the development of five decades, the FDTD method is well-developed and mature now. Except for research, FDTD method can also be used for teaching, especially for the teaching about optoelectronics. FDTD enables us to design, analyze and test modern passive and nonlinear photonic components (such as bio-particles, nanoparticle and so on) for wave propagation, scattering, reflection, diffraction, polarization and nonlinear phenomena. The different FDTD models can help teachers and students solve almost all of the optical problems in optical education. In this paper, we would introduce how to solve scattering problem applying FDTD method and FDTD models. Specifically, the software FDTD solutions has been used for modeling and simulation.

\section{Methods}

According to Yee's scheme, Maxwell curl equation can transform into difference equation and the electromagnetic value can be calculating on the timer shaft.

Firstly, the Maxwell curl equation is shown below:

$$
\left.\begin{array}{l}
\nabla \times H=\varepsilon \frac{\partial E}{\partial t}+\partial E \\
\nabla \times E=-\mu \frac{\partial H}{\partial t}-\sigma_{m} H
\end{array}\right\}
$$

$\mu$ is the permeability; $\mathrm{H}$ is the intensity of magnetic field; is the magnetic permittivity; and is the dielectric constant; $\mathrm{E}$ is the electric field intensity; is the conductivity.

In the rectangular coordinate system, the equation 1 transforms into the equation 2 and equation 3 below:

$$
\begin{aligned}
& \frac{\partial H_{z}}{\partial y}-\frac{\partial H_{y}}{\partial z}=\varepsilon \frac{\partial E_{x}}{\partial t}+\sigma E_{x} \\
& \left.\frac{\partial H_{x}}{\partial z}-\frac{\partial H_{z}}{\partial x}=\varepsilon \frac{\partial E_{y}}{\partial t}+\sigma E_{y}\right\} \\
& \frac{\partial H_{y}}{\partial x}-\frac{\partial H_{x}}{\partial y}=\varepsilon \frac{\partial E_{z}}{\partial t}+\sigma E_{z} \\
& \frac{\partial E_{z}}{\partial y}-\frac{\partial E_{y}}{\partial z}=-\mu \frac{\partial H_{x}}{\partial t}-\sigma_{m} H_{x} \\
& \frac{\partial E_{x}}{\partial z}-\frac{\partial E_{z}}{\partial x}=-\mu \frac{\partial H_{y}}{\partial t}-\sigma_{m} H_{y} \\
& \frac{\partial E_{y}}{\partial x}-\frac{\partial E_{x}}{\partial y}=-\mu \frac{\partial H_{z}}{\partial t}-\sigma_{m} H_{z}
\end{aligned}
$$

For central-difference approximation, Yee has proposed dispersing the space grid and transforming them into rectangular finite difference grid. Make assumption that $\mathrm{X} 、 \mathrm{Y} 、 \mathrm{Z}$ represent the spatial mesh 
size then we get the equation 4 :

$$
(x, y, z)=(i \Delta x, j \Delta y, k \Delta z) .
$$

Also dispersing the time and assume $t$ as the unit of time step then we deduce the equation 5 :

$$
F(x, y, z, t)=F(i \Delta x, j \Delta y, k \Delta z, n \Delta t)=F^{n}(i, j, k) .
$$

Do the central-difference approximation to equation 5 , we obtain the equation $6,7,8$ :

$$
\begin{aligned}
& \frac{\partial F(x, y, z, t)}{\partial x} \approx \frac{F^{n}(i+0.5, j, k)-F^{n}(i-0.5, j, k)}{\Delta x} . \\
& \frac{\partial F(x, y, z, t)}{\partial y} \approx \frac{F^{n}(i, j+0.5, k)-F^{n}(i, j-0.5, k)}{\Delta y} . \\
& \frac{\partial F(x, y, z, t)}{\partial z} \approx \frac{F^{n}(i, j, k+0.5)-F^{n}(i, j, k-0.5)}{\Delta z} .
\end{aligned}
$$

\section{Modeling}

In this paper, we would solve a scattering problem using FDTD method and FDTD models. The example here can be applied in optical education. In this problem, a $\mathrm{Cu}$ nanoparticle has been radiated by unpolarized light, we want to obtain the scattering and absorbing cross section of this scattering phenomenon.

\subsection{Scatter parameters}

For modeling, the size parameters and material should be determined by the particular problems. In this scattering problems, the radius of scatter is $0.05 \mu \mathrm{m}$ and the material of the nanoparticle is $\mathrm{Cu}$. The setting of this scatter in FDTD solutions can be shown in Figure 1.

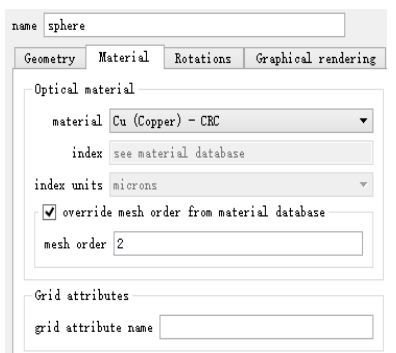

(a)

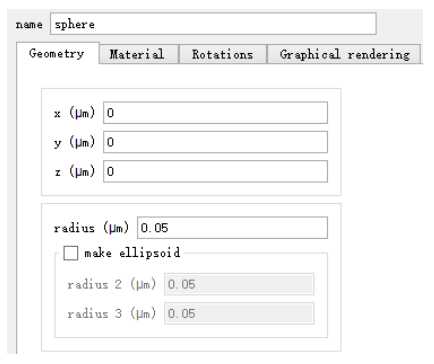

(b)

Fig.1 The setting of sphere (a) the setting of material (b) the setting of size parameters

\subsection{PML boundary condition}

The theory of PML boundary conditions is that setting the special material on the truncated boundary 
and the wave impedance in this special material is totally matched with the wave impedance in the adjacent medium. As a result, the incident wave will pass through the boundary and enter into the PML medium. In this process, the reflection will not happen. So, in FDTD solutions, we choose the PML boundary conditions as shown in Figure 2, whose effect is better than other boundary conditions.

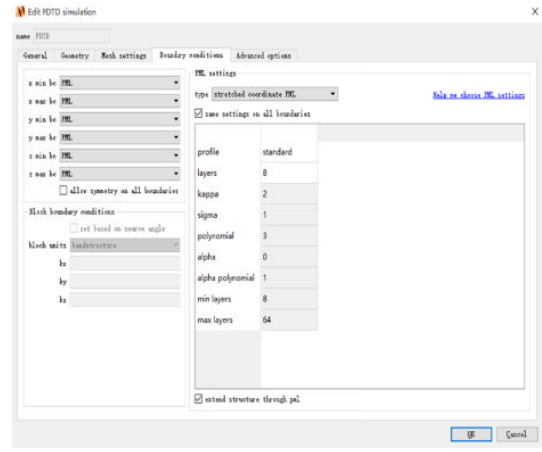

Fig.2 The setting of boundary conditions

\subsection{Mesh accuracy}

To yield accurate results, the grid spacing in the finite difference simulation must be less than the wavelength ${ }^{[3]}$. The stability condition relating the spatial and temporal step size is shown by the equation 9:

$$
c_{\max } \Delta t \leq\left(\frac{1}{\Delta x^{2}}+\frac{1}{\Delta y^{2}}+\frac{1}{\Delta z^{2}}\right)^{-1 / 2}
$$

According to these theory above, we can choose the suitable mesh accuracy in FDTD solutions as shown in Figure 3 below. The minimum requirements of mesh accuracy is that should be less than. For more accurate results, we can choose the option of mesh settings.

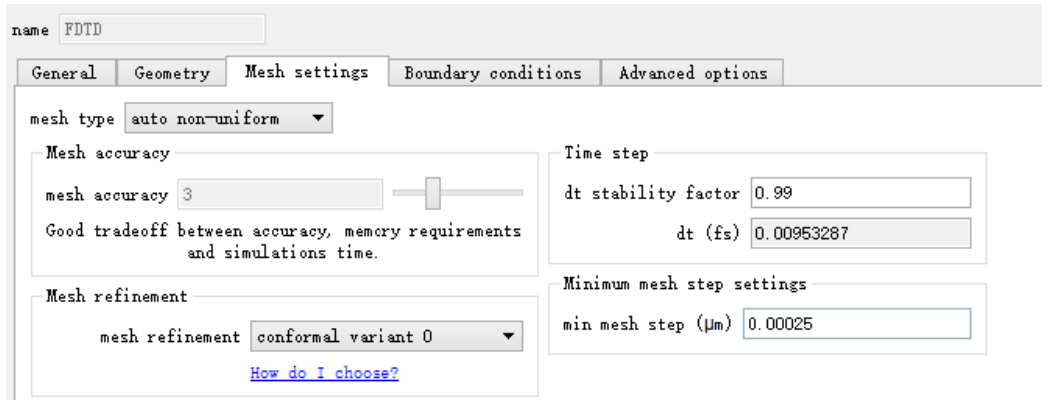

Fig. 3 The setting of mesh accuracy 


\subsection{Total-field scattered-field (TFSF) source}

The simulation region of light scattering can be divided into three parts: scattering object, total field and scattered field ${ }^{[7]}$. The connection boundary is the boundary of total field region and scattered field region; the absorbing boundary is the boundary of the simulation region and the outside space. The division can be shown in Figure 4.

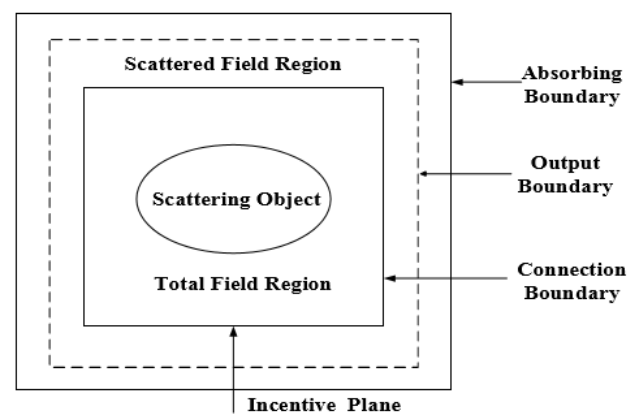

Fig. 4 The division of simulation region

Dealing with scattering problem, placing the excitation source on suitable position is essential. According to the division of simulation region, the excitation source should place on the connection boundary. In this boundary, there are incident wave and scattered wave existing; Out of this boundary, there is only scattered wave existing. In FDTD solutions, choosing the total-field scattered-field (TFSF) source is appropriate to simulate light scattering. In the simulation, the wavelength of excitation source is from $300 \mathrm{~nm}$ to $600 \mathrm{~nm}$ for the results of wide wavelength, the setting of wavelength is shown in Figure 5. And the direction of incidence is forward the $\mathrm{X}$-axis.

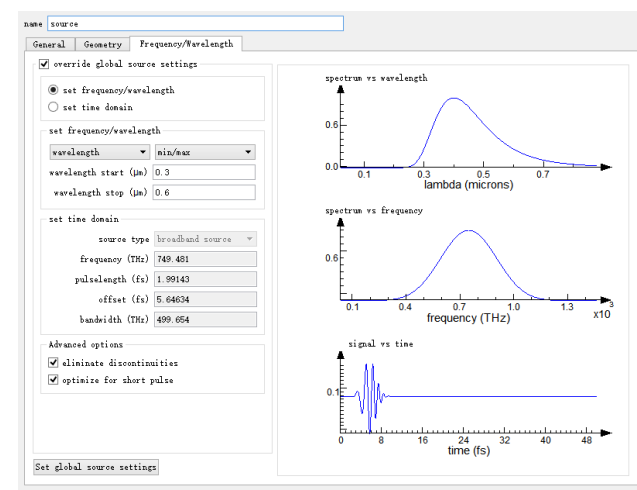

Fig. 5 The setting of wavelength

According to the FDTD method, the FDTD model about scattering from $\mathrm{Cu}$ nanoparticle has been established completely, which is shown in Figure 6. After setting the monitors and analysis group simply in FDTD solutions, the "Run" button can be clicked to perform the simulation. 


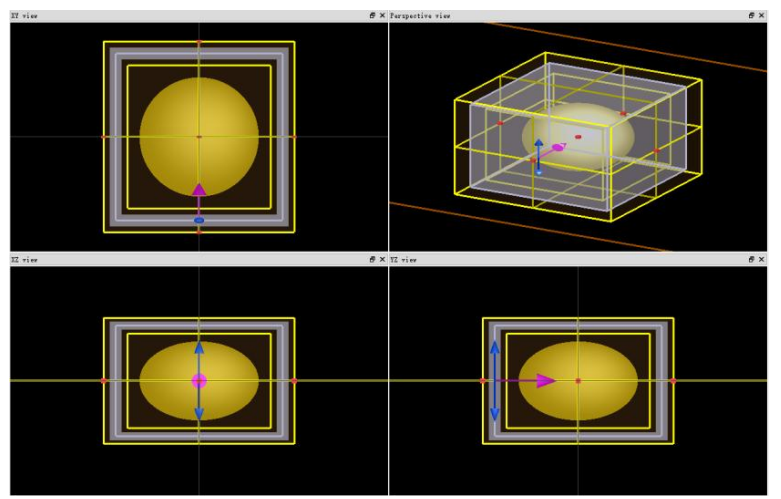

Fig. 6 The FDTD model of light scattering from $\mathrm{Cu}$ nanoparticle

\section{Results}

The absorption cross section (the rate at which energy is removed from the incident plane wave by absorption) is calculated by an analysis group located inside the TFSF source. The analysis group calculates the net power flow into the particle and hence the absorption cross section using the optical theorem. Similarly, the scattering cross section is calculated by an analysis group located outside the TFSF source. This group measures the net power scattered from the particle. The results of absorption cross section and scattering cross section is shown in Figure 7.

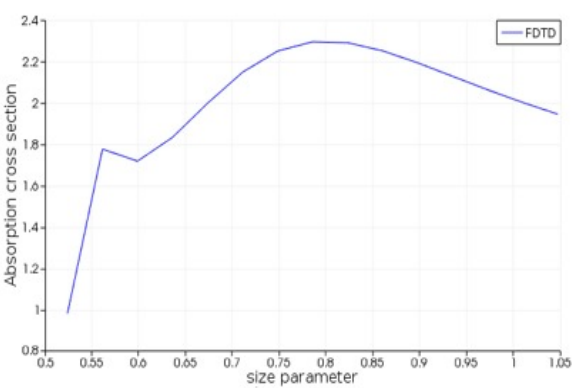

(a)

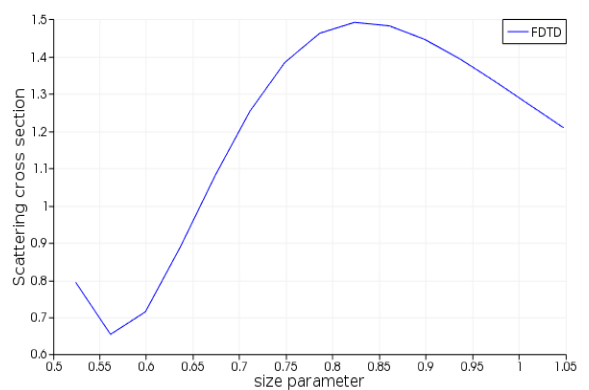

(b)

Fig. 7 The results of simulation (a) the absorption cross section (b) the scattering cross section

\section{Conclusion}

Through modeling and simulation based on FDTD method in FDTD solutions, the scattering problem has been solved easily. Except for scattering problems, the wave propagation, reflection, nonlinear phenomena, radio frequency, CMOS image sensors and OLEDs problems can also be solved by applying FDTD method and FDTD models. The FDTD method and models is simple and easy for teachers and students to study and solve almost all the optical problems. 


\section{Acknowledgments}

This work was supported by National Natural Science Foundation of China (Project no. 6137 7001).

\section{References}

[1] K. S. Yee., "Numerical solution of initial boundary value problems involving maxwell's equations in isotropic media." IEEE Transactions on Antennas \& Propagation. Papers 14(3), 302-307(1966).

[2] C. D. Taylor, D. H. Lam, and T. H. Shumpert., "Electromagnetic pulse scattering in time-varying inhomogeneous media." IEEE Transactions on Antennas and Propagation. Papers 17(5), 585-589(1969).

[3] A. Taflove, and M. E. Brodwin., "Numerical Solution of Steady-State Electromagnetic Scattering Problems Using the Time-Dependent Maxwell's Equations." IEEE Transactions on Microwave Theory \& Techniques. Papers 23(8), 623-630(1975).

[4] G. Mur, "Absorbing Boundary Conditions for the Finite-Difference Approximation of the Time-Domain Electromagnetic-Field Equations." IEEE Transactions on Electromagnetic Compatibility EMC. Papers 23(4), 377-382(2007).

[5] S. S. Zivanovic, K. S. Yee, and K. K. Mei., "A subgridding method for the time-domain finite-difference method to solve Maxwell's equations." IEEE Transactions on Microwave Theory \& Techniques. Papers 39(3), 471-479(2002).

[6] J. P. Renger., "Perfectly matched layer for the FDTD solution of wave-structure interaction problems." IEEE Transactions on Antennas \& Propagation. Papers 44(1), 110-117(1996).

[7] K. Umashankar, and A. Taflove., "A Novel Method to Analyze Electromagnetic Scattering of Complex Objects." IEEE Transactions on Electromagnetic Compatibility. Papers 24(4), 397-405(1982). 